Z Rheumatol 2018 77 (Suppl 1):S1-S3 https://doi.org/10.1007/s00393-018-0473-y

๑) Springer Medizin Verlag GmbH, ein Teil von Springer Nature 2018

CrossMark

\section{U. Müller-Ladner ${ }^{1,2}$}

' Lehrstuhl für Innere Medizin mit Schwerpunkt Rheumatologie, Justus-Liebig Universität Gießen, Gießen Deutschland

${ }^{2}$ Abt. für Rheumatologie und Klinische Immunologie, Kerckhoff-Klinik GmbH, Bad Nauheim, Deutschland

\title{
Rheumatologische Forschungsnetzwerke in Deutschland
}

Kaum eine andere Disziplin beinhaltet so viele Ideen zu Forschungsthemen wie die Rheumatologie und Klinische Immunologie. Dies v. a. aufgrund der hohen Variabilität des Immunsystems, dessen ubiquitärer Präsenz im gesamten Organismus und der Beteiligung an nahezu allen klinisch normalen und abnormalen Situationen. Viel schwieriger ist die Umsetzung dieser Ideen in konkrete Projekte, sowohl als Einzelprojekt, aber auch im Verbund mit anderen Kollegen.

Diese Problematik ist im Wesentlichen der Tatsache geschuldet, dass an den deutschen Universitäten und Universitätskliniken die Fächer Rheumatologie und klinische Immunologie bekanntermaßen eher dürftig vertreten sind. Umso höher ist es zu bewerten, dass es in den vergangenen Jahren gelungen ist, auf nationaler Ebene bei den verschiedenen öffentlichen Förderinstitutionen, v. a. der Deutschen Forschungsgemeinschaft und des Bundesministeriums für Bildung und Forschung, aber auch auf Eigeninitiative ohne Förderung und mit Unterstützung der Kollegen innerhalb der Deutschen Gesellschaft für Rheumatologie und benachbarten Disziplinen große, raum- und themenübergreifende Forschungskonsortien aufzustellen und diese $\mathrm{zu}$ wegweisenden Resultaten $\mathrm{zu}$ führen.

Diese Forschungsnetzwerke und ihre Ergebnisse werden sehr eindrucksvoll in den nachfolgenden Beiträgen vorgestellt und illustrieren sehr deutlich die enorme wissenschaftliche Schlagkraft der beteiligten Kollegen, aber auch die hierfür notwendige harmonische wissenschaftliche Zusammenarbeit innerhalb der Deutschen Gesellschaft für Rheumatologie und den kooperierenden Disziplinen.

Ein Paradebeispiel, welches auch sehr eindrucksvoll zeigt, wie Kollegen bundesweit vernetzt werden können, ist das Schwerpunktprogramm Immunobone der Deutschen Forschungsgemeinschaft, welches die Funktionen des Immunsystems am und im Knochenmark v. a. bei chronisch entzündlichen Erkrankungen untersucht hat. Wie in mehreren Beiträgen in diesem Heft der Zeitschrift für Rheumatologie sehr anschaulich aufgezeigt wird, konnte das Konsortium zeigen, dass die Funktion des Knochenmarks hinsichtlich chronisch entzündlicher Erkrankungen weit über die Primärfunktion der Blutbildung hinausgeht. Hierbei ist eines der zentralen Erkenntnisse, dass die Veränderung der zellulären Zusammensetzung des Knochenmarks und deren Botenstoffe die Funktion des Immunsystems signifikant beeinflussen kann. Umgekehrt können auch chronisch entzündliche Erkrankungen einen deutlichen Einfluss auf die Funktionen des Knochenmarks nehmen. Hieraus folgte auch, dass den Lebensstil modifizierbare Faktoren die Immunfunktion des Organismus über das Knochenmark mit beeinflussen können.

Insbesondere die Interaktion zwischen Komponenten der zellulären und azellulären Knochenstruktur und des Immunsystems war ein zentraler Ansatzpunkt des Schwerpunktprogrammes Immunobone. Im Laufe der 6-jährigen
Förderperiode konnte mit diesem Ansatz unter anderem gezeigt werden, dass eine direkte Verbindung zwischen Autoimmunität und Knochendestruktion über die Interaktion von citrullinierten Peptiden und der Osteoklastendifferenzierung besteht. Daneben konnten zahlreiche Signalmoleküle der Zytokinreihe direkte Effekte auf knochenabbauende Signalwege zugeordnet werden. Selbst für „vergessene“ Zellen wie den Mastzellen, deren Bedeutung für die Pathogenese der Gelenkentzündung kontinuierlich kontrovers diskutiert wird, konnten klarere pathophysiologische Zuordnungen erarbeitet werden. Vice versa ermöglichten die Experimente auch die Herausarbeitung von körpereigenen Regulationsmechanismen zum Schutz vor Überaktivität des Immunsystems. Hier standen v. a. die natürlich vorkommenden regulatorischen T-Lymphozyten und deren Interaktion mit ossären Substanzen im Mittelpunkt. Daneben rückten auch die nicht nur bei bakteriell induzierten Knochenentzündungen aktiven Neutrophilen und deren Einfluss in den Mittelpunkt. Grund hierfür war deren Rolle bei der Bildung der sog. „neutrophil extracellular traps“ im Rahmen einer chronischen Entzündung hinsichtlich des Verlaufs der Entzündung und des resultierenden Knochenumbaus. Ein weiterer Baustein betraf die Regulation des Knochens und der Knochenstoffwechselwege durch die entzündliche Aktivität der Grunderkrankung. Hier wurde v. a. die Schutzregulation von Glukokortikoiden und deren Rezeptoren bei der Entzündungsauflösung 


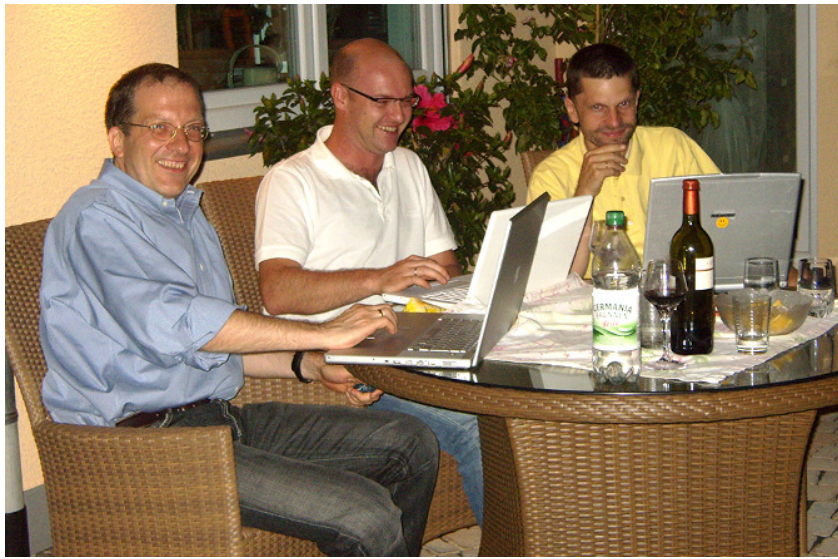

Abb. 1 Initiation des Immunobone-Antrags. (Aufnahme: Thomas Pap)

untersucht - stets verbunden mit dem langfristigen Problem des zu aktiven Knochenabbaus, welcher in einer zwar entzündungsfreien, aber dann doch für den Körper belastenden Osteoporose resultieren kann.

Im Gegensatz $\mathrm{zu}$ den thematisch eher offenen Forschungsförderungen der Deutschen Forschungsgemeinschaft sind BMBF-geförderte Programme regelhaft themenorientiert. Auch hier konnte die rheumatologische Forschergemeinschaft einen Schwerpunktverbund einwerben. Der Arthromark benannte Verbund hatte die Aufgabe, klinisch geeignete Biomarker zur Frühdiagnostik und möglicherweise auch zur Vorhersage des Therapieerfolgs $\mathrm{zu}$ suchen und $\mathrm{zu}$ identifizieren.

Hierzu war es notwendig, die Voraussetzung zu schaffen, dass viele große Daten bzw. Datenbanken zusammenarbeiten, um mithilfe einer geeigneten Biometrie trotz der hohen Variabilität der Grunderkrankung doch für die klinisch nutzbare Realität geeignete Biomarker zu finden und zu etablieren. Obwohl es nahezu unmöglich ist, in einer so kurzen Zeit all diese Fragen zu beantworten, konnte der Verbund dennoch zahlreiche wegweisende Ergebnisse herausarbeiten. Diese betrafen die Galaktosylierung und deren Beeinflussung durch therapeutische Ansätze bei der rheumatoiden Arthritis, die Herausarbeitung von benennbaren genetischen Markern, die für den Verlauf bedeutsam sein könnten, den ersten Nachweis von Proteinbiomarkern durch Einsatz von Transkriptomanalysen und bestimmte Autoantikör- perprofile bei chronisch entzündlichen Gelenkerkrankungen.

Selbst die eher schwierig zugänglichen Spondylarthritiden erlaubten durch die Zusammenarbeit der Kollegen eine Herausarbeitung von Biomarkern von potenziell prädiktiver Bedeutung. Letzteres wurde unterstützt durch die neu entwickelten hochauflösenden bildgebungstechnischen Methoden, die nach und nach in die klinische Rheumatologie Eingang finden. All dies wäre nicht möglich gewesen, wenn nicht eine entsprechende Bioinformatik zusammen mit zugänglichem Biobanking dem Konsortium zur Verfügung gestanden hätte.

Einem ganz anderen Themenfeld widmete sich der MSK-Forschungsverbund Neuroimmunologie in Schmerz (NeuroIMPA), welcher die Beziehung zwischen dem Immunsystem und dem Nervensystem bei muskuloskeletalen Erkrankungen insbesondere hinsichtlich der Entstehung von Schmerzen und Verlauf von Frakturheilungen und Arthritiden untersuchte. Die Besonderheit dieses Verbundes ist, dass sonst 2 klinisch eher weniger etabliert kooperierende Wissenschaftsbereiche, die Immunologie und die Neurologie - insbesondere hinsichtlich entzündlich rheumatischer Erkrankungen - sich gemeinsam der Aufgabe widmeten, welche neurologischen Veränderungen einen Einfluss auf Entzündungsvorgänge nehmen könnten und welche pathophysiologischen Stoffwechselwege hierbei zugrunde liegen.

Das Konsortium konnte sehr eindrucksvoll zeigen, dass proinflammatorische Zytokine nicht nur an der
Schmerzentstehung und Perpetuation beteiligt sind, sondern auch durch Bildung von Opiodpeptiden direkt in die Schmerzwahrnehmung eingreifen können. Interessanterweise war nicht nur der Schmerz diesen Signalmolekülen zugeordnet, sondern auch Veränderungen in Knochenneu- und -umbildung. Insofern war es ein wegweisendes Ergebnis dieses Konsortiums, dass die Modulation neuronaler Mechanismen in die Krankheitsverläufe muskuloskeletaler Erkrankungen direkt einbezogen ist und zahlreiche Ansätze für therapeutische Ideen der Zukunft bietet.

Auch wenn es vielleicht banal klingen mag, aber eine gut durchdachte und ausgeführte klinische Studie zum Nachweis eines therapeutischen Effekts gehört zu den relevantesten, aber gleichzeitig am schwierigsten einzuwerbenden Drittmitteln, da diese aus Eigenmitteln schwer für Kliniken und Universitäten zu stemmen ist und die Kompetition im exzellent hierfür maßgeschneiderten Programm klinische Studien der DFG und des BMBF mit einer Förderquote von unter $10 \%$ aufgrund des hohen Antragseingangs zu den am schwierigsten zu erreichenden Einwerbungen gehört.

Trotzdem kann dieses auch in der klinischen Rheumatologie gelingen, wie die Rheumaforschungsgruppe Ruhr, die aus Fachärzten des Rheumazentrums Ruhrgebiet aus Klinik und niedergelassenen Kollegen besteht, eindrucksvoll gezeigt hat, indem sie eine solche Einwerbung zur Frage der optimalen Kortikoiddosis bei der Behandlung der rheumatoiden Arthritis realisierte.

Die detaillierten Ergebnisse hierzu werden 2018 mit Spannung erwartet. Des Weiteren gelang es dieser Gruppe, im Rahmen einer Förderung des Innovationsfonds zusammen mit Krankenkassen eine weitere tägliche Problematik zu untersuchen: inwieweit die Delegation ärztlicher Leistungen an geschulte rheumatologische Fachassistenten einen Vorteil in der Versorgung erbringt. Auch diese Studie ist naturgemäß langfristig angelegt, und im Jahr 2020 werden erste Ergebnisse hierzu vorliegen. Diese klinische Forschergruppe in einem regional begrenzten Gebiet illustriert auch, wie viele verschiedene und in diesem Heft 
der Zeitschrift für Rheumatologie weiter angeführte Projekte bei entsprechender Motivation der Kollegen, die in der Regel unter Rheumatologen unbeschränkt vorhanden ist, in ,relativ kurzen“ Zeiträumen erfolgreich durchgeführt werden können.

In Zusammenschau aller hier vorgestellten Forschungsverbünde unterschiedlichster Couleur ist zu betonen, dass absolut kein Grund besteht, wissenschaftliche Aktivitäten im Bereich der Basisforschung und der klinischen Forschung zu zurückhaltend zu verfolgen, nur weil nicht an jedem universitären Standort ein Lehrstuhl besteht. Die auf den folgenden Seiten vorgestellten Forschungsprojekte sollten eher dazu motivieren, alle interessierten Kollegen anzusprechen, um in kleiner und größerer Gemeinschaft aktuelle Fragestellungen in die Tat und die Ergebnisse für die klinische Zukunft umzusetzen - die Fördermittel sind hierfür im Förderatlas der Drittmittelgeber von Deutschland auf jeden Fall vorhanden, und es gilt die altbekannte Weisheit: Für die Realisierung einer pfiffigen Idee braucht es in der Regel am Anfang nur eine laue Sommernacht und einige Gläser guten Wein (• Abb. 1).

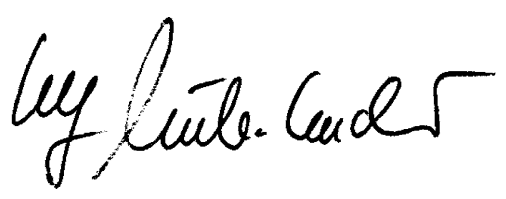

Prof. Dr. med. Ulf Müller-Ladner

\section{Korrespondenzadresse}

\section{Prof. Dr. med. U. Müller-Ladner} Abt. für Rheumatologie und Klinische Immunologie, Kerckhoff-Klinik GmbH Benekestr. 2-8, 61231 Bad Nauheim, Deutschland

U.Mueller-Ladner@kerckhoff-klinik.de

Interessenkonflikt. U. Müller-Ladner gibt an, dass kein Interessenkonflikt besteht. 